Research Article

\title{
Chaotic Dynamics and Chaos Control of Hassell-Type Recruitment Population Model
}

\author{
Guo Feng \\ School of Data and Computer Science, Shandong Women's University, Jinan, China 250300 \\ Correspondence should be addressed to Guo Feng; sdwugf@163.com
}

Received 23 January 2020; Revised 4 March 2020; Accepted 11 March 2020; Published 9 April 2020

Academic Editor: Nikos I. Karachalios

Copyright (c) 2020 Guo Feng. This is an open access article distributed under the Creative Commons Attribution License, which permits unrestricted use, distribution, and reproduction in any medium, provided the original work is properly cited.

\begin{abstract}
For certain parameters, the mapping of a Hassell-type recruitment population model has a chaotic attractor. The control parameter is disturbed slightly with time by the improvement OGY method. When the mapping point wanders to the neighborhood of the periodic point, the control parameter is perturbed. The chaotic motion is controlled on the stable periodic period-1 point and period- 2 orbits, and the influence of different control parameter ranges on the control average time is analyzed. When the selected regulator poles are different, the number of iterations used to control chaotic motion on a stable periodic orbit is different. Numerical simulations are presented to illustrate our results with the theoretical analysis and show the effect of the control method.
\end{abstract}

\section{Introduction}

Leslie models [1] with nonlinear fertility and mortality can have complicated dynamical behavior. Ugarcovici and Weiss [2] studied the Ricker model. This model is described by the two-dimensional mapping $R_{a, b}: \mathbb{R}_{+}^{2} \longrightarrow \mathbb{R}_{+}^{2}$ :

$$
R_{a, b}(x, y)=\left((a x+\gamma a y) \mathrm{e}^{-\lambda(x+y)}, b x\right),
$$

where $x$ and $y$ stand for the density of the first age group and the second age group. $a$ and $\gamma a$ are the group's initial fertility rates $(a, \gamma>0), b$ is the survival rate from the first age group to the second one, and $\lambda$ is the decay index, $\lambda>0$. In equation (1), the fertility rate monotonically decreases as a function of the total population size, and the fertility decay is exponential. The other model is Hassell model. It is described by the two-dimensional mapping $H_{a, b}: \mathbb{R}_{+}^{2} \longrightarrow \mathbb{R}_{+}^{2}$ :

$$
H_{a, b}(x, y)=\left((a x+\gamma a y)(1+x+y)^{-\beta}, b x\right),
$$

where $a$ and $\gamma a$ are the group's initial fertility rates, $b$ is the survival rate from the first age group to the second one, and $\beta$ is the decay index, $\beta>1$. In equation (2), the fertility rate monotonically decreases as a function of the total population size, and the fertility decay is polynomial.
For some parameter values, these models admit an ergodic attractor which supports a unique physical probability measure. This physical measure satisfies in the strongest possible sense the population biologist's requirement for ergodicity in their population models. The Lebesgue measure basin is the whole plane $\mathbb{R}_{+}^{2}$; Hassell [3], Wikan and Mjølhus [4], and Ugarcovici and Weiss [2] showed that Ricker mapping and Hassell mapping produce Hénon-like chaotic attractors.

Here, we present a procedure to contend chaotic dynamics in a Hassell-type recruitment population model. As a is between 0 and $60, b=0.7, \gamma=0.8$, and $\beta=22$, Hassell mapping shows rich chaotic dynamic behavior. In biology or ecology, the complex chaotic behavior of this mapping shows the relationship between the number, birth rate, and survival rate in a population, whether it survives in a balanced state or makes the population develop in disorder or chaos. This research can provide theoretical basis and help for the research in biology or ecology. For example, it is applied in marine fishing or in the reproduction and population growth of a certain species in nature.

The study of chaos control began in the late 1980s. The early research idea is to use the existing dynamic control strategies and destroy the conditions for the occurrence of 
chaotic motion. Ott et al. [5] proposed a method to control chaos. The basic idea of controlling chaos can be understood by considering the following one-dimensional logistic map, one of the best studied chaotic systems:

$$
x_{n+1}=f\left(x_{n}, r\right)=r x_{n}\left(1-x_{n}\right) \text {, }
$$

where $x$ is restricted to the unit interval [ 01 1] and $r$ is a control parameter. The logistic map in the neighborhood of a periodic orbit can be approximated by a linear equation expanded around the periodic orbit. Denote the target period- $m$ orbit to be controlled as $x(i), i=1,2, \ldots, m$, where $x(i+1)=f(x(i))$ and $x(m+1)=x(1)$. Assume that, at time $n$, the trajectory falls into the neighborhood of component $i$ of the period- $m$ orbit. The linearized dynamics in the neighborhood of component $(i+1)$ is

$$
\begin{aligned}
x_{n+1}-x(i+1)= & \frac{\partial f}{\partial x}\left[x_{n}-x(i)\right]+\frac{\partial f}{\partial r} \Delta r_{n} \\
= & r_{0}[1-2 x(i)]\left[x_{n}-x(i)\right] \\
& +x(i)[1-x(i)] \Delta r_{n},
\end{aligned}
$$

where the partial derivatives are evaluated at $x=x(i)$ and $r=r_{0}$. To make $x_{n+1}$ stay in the neighborhood of $x(i+1)$, let $x_{n+1}-x(i+1)=0$, which gives

$$
\Delta r_{n}=r_{0} \frac{[2 x(i)-1]\left[x_{n}-x(i)\right]}{x(i)[1-x(i)]} .
$$

Equation (5) holds only when the trajectory $x_{n}$ enters a small neighborhood of the period- $m$ orbit, i.e., when $\left|x_{n}-x(i)\right| \ll 1$, and hence, the required parameter perturbation $\Delta r_{n}$ is small. Let the length of a small interval defining the neighborhood around each component of the period- $m$ orbit be $2 \varepsilon$. In general, the required maximum parameter perturbation $\delta$ is proportional to $\varepsilon$. Since $\varepsilon$ can be chosen to be arbitrarily small, $\delta$ can also be made arbitrarily small. The average transient time before a trajectory enters the neighborhood of the target periodic orbit depends on $\varepsilon$ (or $\delta)$. When the trajectory is outside the neighborhood of the target periodic orbit, any parameter perturbation is not applied, so the system evolves at its nominal parameter value $r_{0}$. Hence, we set $\Delta r_{n}=0$ when $\Delta r_{n}>\delta$. The parameter perturbation $\Delta r_{n}$ depends on $x_{n}$ and is time-dependent.

Pyragas [6], Shinbrot et al. [7], Pyragas [8], and Kocarev and Parlitz [9] have put forward different improvement measures according to various situations and further developed the OGY method, which has laid a good foundation for the application of chaos. Flipe et al. [10] adopt the pole assignment technique in system control to further improve the OGY method. The chaos of high periodic states and high-dimensional dynamic systems in chaotic attractors is controlled by the improved OGY method.

By the OGY method, Guo et al. $[11,12]$ studied the chaos control of two-dimensional Lauwerier mapping

$$
\left\{\begin{array}{l}
x_{n+1}=b x_{n}\left(1-2 y_{n}\right)+y_{n} \\
y_{n+1}=a y_{n}\left(1-y_{n}\right)
\end{array}\right.
$$

and a piecewise linear Lozi mapping

$$
\left\{\begin{array}{l}
x_{n+1}=1-a\left|x_{n}\right|+y_{n} \\
y_{n+1}=b x_{n}
\end{array}\right.
$$

The chaotic motions of equations (6) and (7) are both controlled on periodic- 1 and periodic- 2 orbits. Guo et al. [13] studied the chaos control of two-degree-of-freedom collision vibration system by the OGY method. A four-dimensional discontinuous system is studied. The chaotic motion is controlled on periodic- 1 and periodic- 2 orbits.

The Hassell mapping produces a chaotic attractor as $a=31, b=0.7, \gamma=0.8$, and $\beta=22$. The unstable period -1 point and unstable period- 2 points are controlled on the periodic orbits.

\section{The Hassell Recruitment Population Model}

The Hassell recruitment population model has two generations [3]. It is described by the two-dimensional mapping $H_{a, b}: \mathbb{R}_{+}^{2} \longrightarrow \mathbb{R}_{+}^{2}$ :

$$
H_{a, b}(x, y):\left\{\begin{array}{l}
f(x, y)=(a x+\gamma a y)(1+x+y)^{-\beta} \\
g(x, y)=b x
\end{array}\right.
$$

where $x$ and $y$ stand for the density of the first age group and the second one. $a$ and $\gamma a$ are the fertility rates of the group at time zero, $b$ is the survival rate from the first age group to the second one, and $\beta$ is the decay index, $\beta>1$. When $\beta=1$, the case corresponds to the model of Beverton and Holt [14]. The attractors are fixed points.

For the parameters $\gamma=0.8$ and $\beta=22$ and fixed survival rate $b=0.7$, when the initial fertility rate $a$ changes between 0 and 35 , equation (8) generates complicated features, shown in Figure1(a)-1(e). For $0<a<0.455,(0,0)$ is a global attractor. There exists a positive fixed point that is asymptotically stable for $0.455<a<7$. For $a$ between 7 and 10 , unstable coexistence between the first age group and the second one can be observed, with chaotic bands having narrow and wide periodic windows. Subsequently, a cascade of period-halving is shows in Figure 1(b). For $a$ between 10 and 15 , the system goes through a quasi-periodic region (Hopf bifurcation occurs at $a=12.5$, including frequency lockings which appear as a collapse of the invariant circle to a periodic orbit), beyond which equation (8) coexists in a stable manner for a large range of the parameter $a$ (as shown in Figure 1(c)). The system enters quasi-periodicity again when $a=15$. For $a$ between 15 and 17, it coexists in an unstable manner again, with chaotic bands having narrow and wide periodic windows. For $a$ between 17 and 25, Hopf bifurcation occurs again (as shown in Figure 1(d)). For $a$ between 25 and 29, the bifurcation diagram is a periodic window with a periodic attractor, but that it includes ambiguous dynamic behavior. Several attractors coexist in this region (as shown in Figure 1(e)). For a greater than 31, a chaotic attractor appears. A Hénon chaotic attractor [15] coexists with an attracting invariant curve (as shown in Figure 2). For $a$ between 0 and 60, the global bifurcation is shown in Figure 1(a).

The largest Lyapunov exponent measures the average exponential rate of divergence or convergence of nearby 


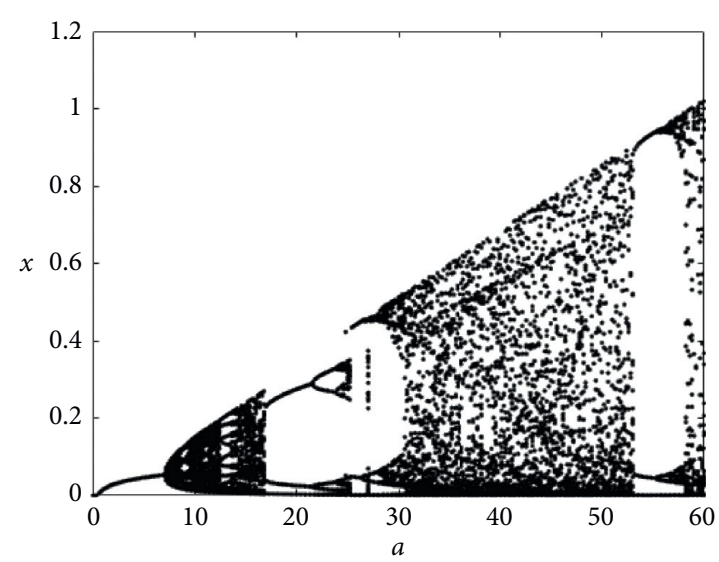

(a)

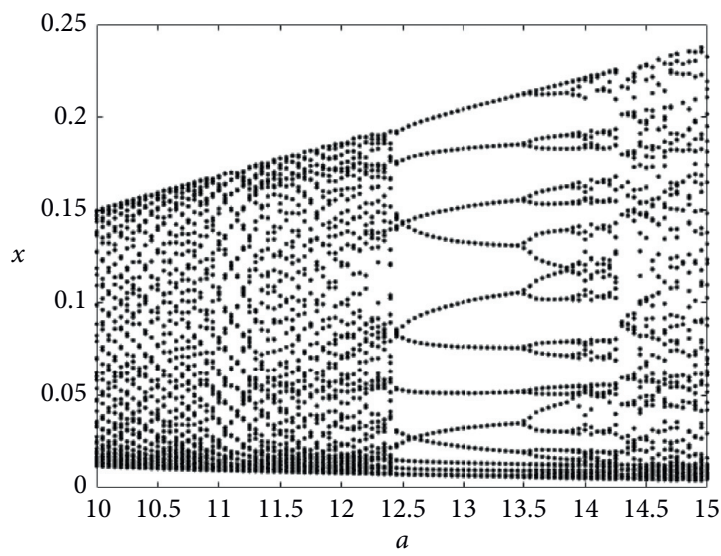

(c)

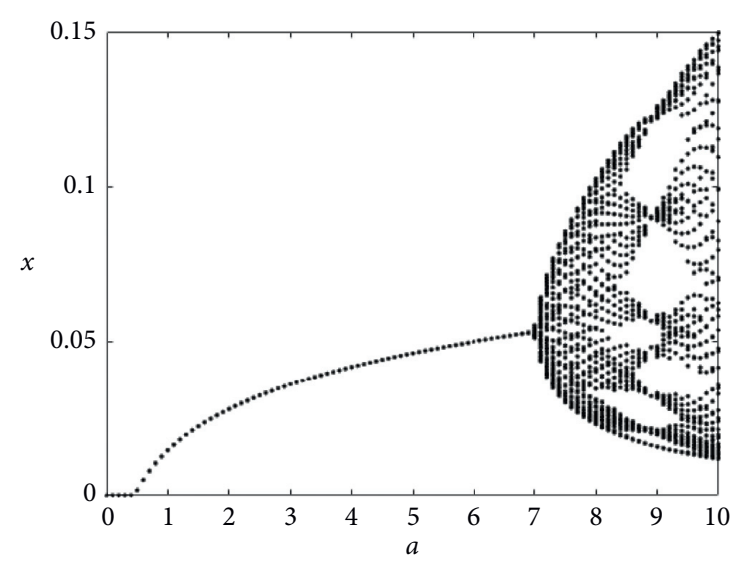

(b)

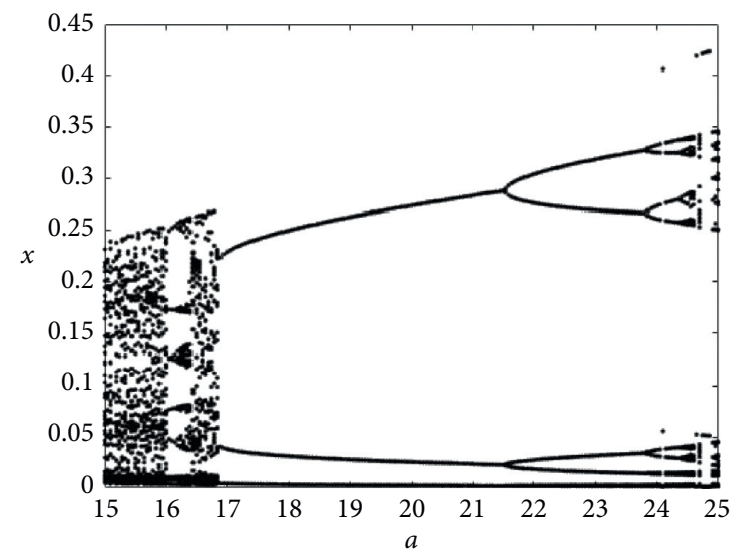

(d)

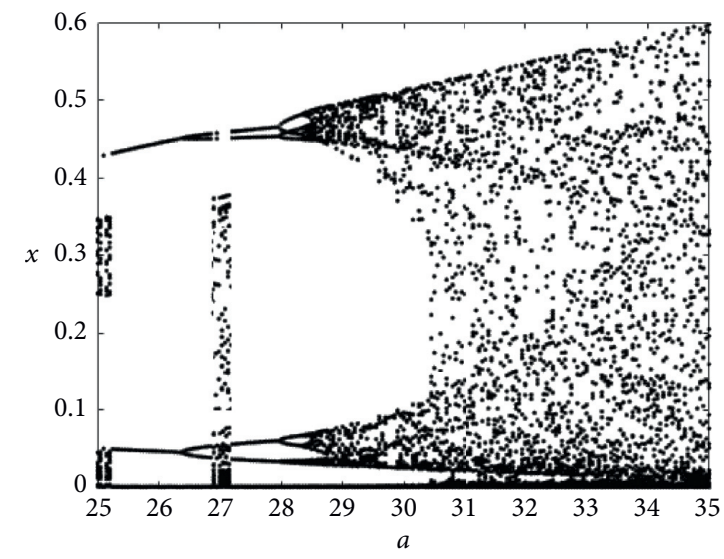

(e)

Figure 1: Bifurcation diagrams of the Hassell mapping $(\gamma=0.8, \beta=22, b=0.7$, and $0<a<35)$.

orbits in the phase space. Whenever two nearby orbits move too far apart, one of the orbits has to be moved back to the vicinity of the other along the line of separation. For a chaotic attractor, the largest Lyapunov exponent must be positive. If the largest Lyapunov exponent is negative, this implies a stable state or a periodic attractor. The largest
Lyapunov exponents of the Hassell mapping have been calculated and plotted in Figure 3. When $0<a<15$, the largest Lyapunov exponent is negative. When $15<a<30$, the largest Lyapunov exponent can be negative or positive, and the system has Hopf bifurcation and enters quasi-periodicity and at last generates the chaotic state. When $a>30$, the 


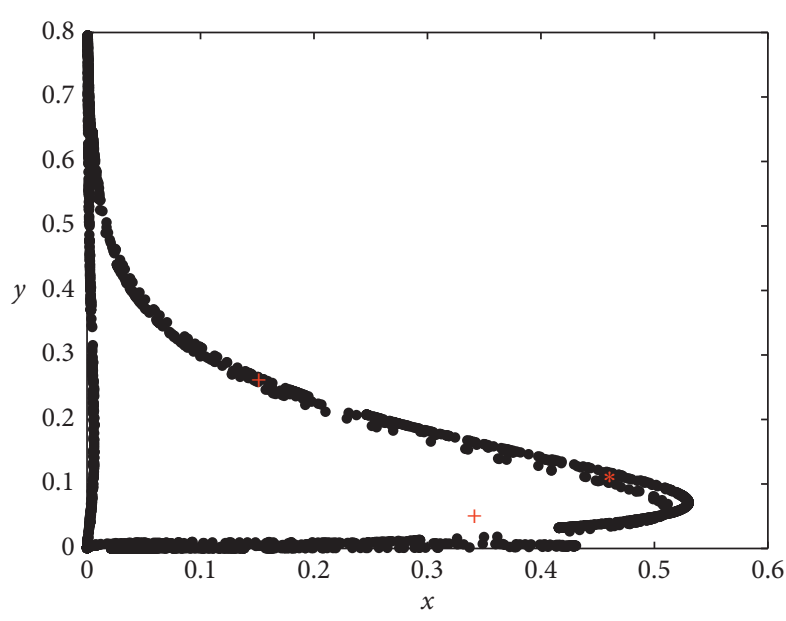

(a)

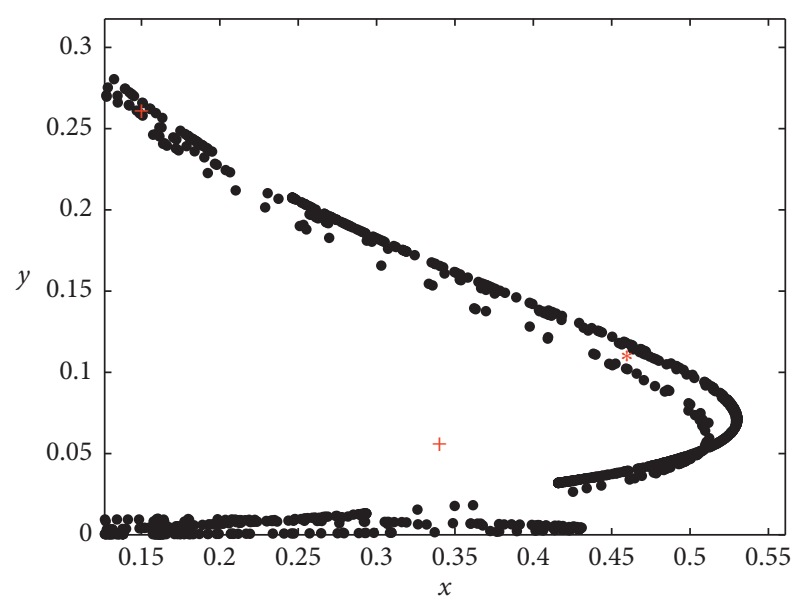

(b)

FIgURE 2: The chaotic attractor of the Hassell mapping with unstable periodic orbits of period-1(.) and period-2(+).

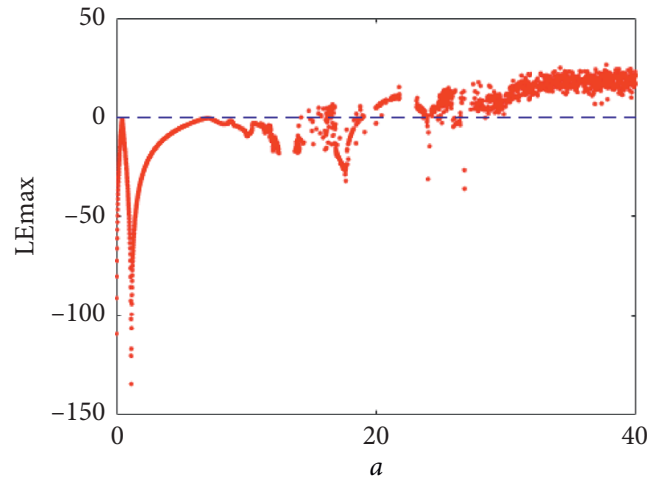

(a)

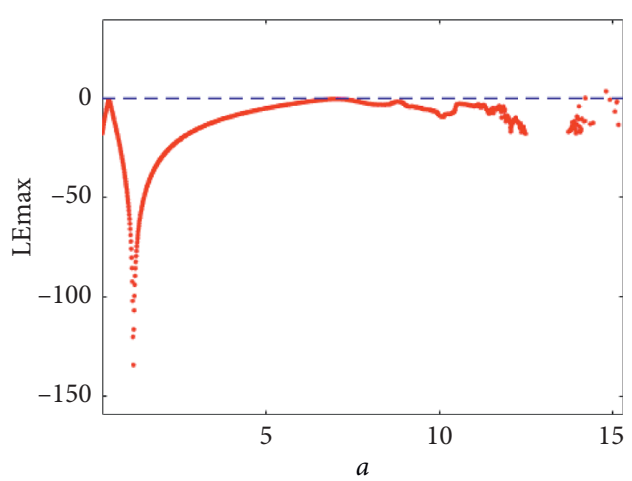

(b)

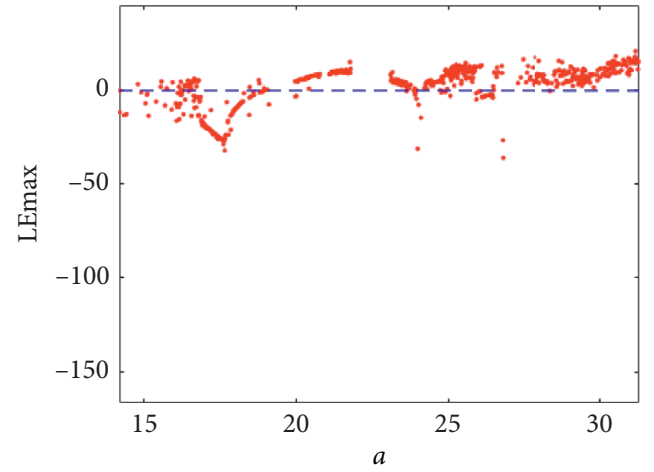

(c)

FIGURE 3: Lyapunov exponent of Hassell mapping $(\gamma=0.8, \beta=22, b=0.7$, and $0<a<40)$.

largest Lyapunov exponent is positive. The dynamics behavior is chaotic. And the chaotic attractor is found as LE $\max =2.1392 \mathrm{e}^{0.3}$.

\section{Pole Assignment Technique for Controlling Chaos}

The system is first written in the form of a discrete time [10]:

$$
Z_{i+1}=F\left(Z_{i}, a\right), \quad Z_{i} \in R^{2}, a \in R
$$

$F$ is sufficiently smooth and $a$ is an externally adjustable real parameter. That is, it requires $|a-\bar{a}|<\delta$ at some time, and $\bar{a}$ is a rated value. It is assumed that there is a chaotic attractor of equation (9) for $a=\bar{a}$. Now, the aim is to change the parameters such that the chaotic attractor involves almost all of the initial conditions so that the dynamic 
behavior of the system converges to the desired periodic orbit in the attractor. By the OGY method, due to the ergodicity of the chaos dynamics, when the state trajectory enters the vicinity of the unstable periodic orbit to be stabilized, a feedback control law is applied to control the trajectory to move to the desired unstable periodic orbit.

If $Z_{*}(\bar{a})$ is the unstable fixed point, by first-order Taylor expansion, equation (9) can be written as

$$
Z_{i+1}-Z_{*}(\bar{a})=A\left(Z_{i}-Z_{*}(\bar{a})\right)+B(a-\bar{a}) .
$$

Find out the values of the matrices $A$ and $B$ at $Z=Z_{*}(\bar{a})$ and $a=\bar{a}$, where $A$ is the partial derivative matrix of $F(z, a)$ to $z, A=D_{z} F(z, a)$, and $B$ is the partial derivative matrix of $F(z, a)$ to $a, B=D_{a} F(z, a)$. The time-dependent control parameter $a$ is in the form of a linear function with respect to the variable

$$
a-\bar{a}=-K^{T}\left(Z_{i}-Z_{*}(\bar{a})\right) .
$$

Replace equations (11) into (10):

$$
Z_{i+1}-Z_{*}(\bar{a})=\left(A-B K^{T}\right)\left(Z_{i}-Z_{*}(\bar{a})\right) .
$$

So, as long as the matrix $A-B K^{T}$ is asymptotically stable, that is, if the modulus of its eigenvalues is less than 1 , the fixed point $Z_{*}(\bar{a})$ is stable. The following key questions is how to determine the matrix $K^{T}$, which can stabilize the chaotic motion at a stable periodic point. The pole assignment is solved according to [16]. The matrix $C_{n \times n}$ is a controllable matrix, and the rank is $n$ :

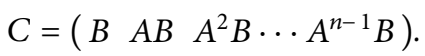

The solution of pole assignment is given by $K^{T}=\left(\alpha_{n}-a_{n}, \ldots, \alpha_{1}-a_{1}\right) T^{-1}$, where $T=C W, W$ is a matrix of order $n$ :

$$
\begin{aligned}
& W=\left(\begin{array}{ccccc}
a_{n-1} & a_{n-2} & \ldots & a_{1} & 1 \\
a_{n-2} & a_{n-3} & \ldots & 1 & 0 \\
\ldots & \ldots & \ldots & \ldots & \ldots \\
a_{1} & 1 & \ldots & 0 & 0 \\
1 & 0 & \ldots & 0 & 0
\end{array}\right), \quad(14) \\
& A=\left(\begin{array}{c}
a(1+1 x-\beta x+y-\beta \gamma y)(1+x+y)^{-\beta-1} \\
a(\gamma+\gamma x-\beta x+\gamma y-\gamma y \beta)(1+x+y)^{-\beta-1} \\
b
\end{array}\right)_{\left(x_{*}, y_{*}\right)}=\left(\begin{array}{cc}
-0.010-0.011 \\
1.5 & 0
\end{array}\right), \\
& B=\left.\left(\begin{array}{c}
\left((x+\gamma y)(1+x+y)^{-\beta}\right) \\
0
\end{array}\right)\right|_{\left(x_{*}, y_{*}\right)}=\left(\begin{array}{c}
1.44 \\
0
\end{array}\right) .
\end{aligned}
$$

where $a_{i}(i=1, \ldots, n)$ are the coefficients of the characteristic polynomial of the matrix $A$, that is,

$$
\operatorname{det}(\lambda I-A)=\lambda^{n}+a_{1} \lambda^{n-1}+\cdots+a_{n}
$$

and $\alpha_{1}, \alpha_{2}, \ldots, \alpha_{n}$ are the coefficients of the characteristic polynomial $\operatorname{det}\left(A-B K^{T}\right)$, that is,

$$
\operatorname{det}\left(s I-\left(A-B K^{T}\right)\right)=s^{n}+\alpha_{1} s^{n-1}+\cdots+\alpha_{n} .
$$

After working out $K^{T},\left|K^{T}\left(Z_{i}-Z_{*}(\bar{a})\right)\right|<\delta$ is obtained by $|a-\bar{a}|<\delta$ and equation (12).

\section{The Chaos Control of Hassell Mapping}

As shown in Figures 2(a) and 2(b), for $\gamma=0.8, \beta=22$, and $b=0.7$, when $a=31$, the dynamic has period-1 point and period-2 points. The dynamic behavior of the mapping is chaotic at $\bar{a}=31$. At the same time, there is a chaotic attractor, which is the closure of the unstable manifolds of the saddle points. And there is an infinite number of unstable periodic orbits in the chaotic attractor. There are an unstable fixed point and two unstable period-2 points embedding in the chaotic attractor.

4.1. Control of Period-1 Point of Hassell Mapping. Let the control parameter $a$ be a variable near the rated value $\bar{a}=31$ at $\gamma=0.8, \beta=22$, and $b=0.7$, By $f(x, y)=x$ and $g(x, y)=y$, the fixed point is $\left(x_{*}, y_{*}\right)=(0.46,0.11)$. Bring the fixed point into the matrixes $A$ and $B$ :
A controllable matrix is a matrix with rank 2 as

$$
C=\left(\begin{array}{ll}
B & A B
\end{array}\right)=\left(\begin{array}{cc}
1.44 & -0.144 \\
0 & 2.16
\end{array}\right) \text {. }
$$

The solution of the pole assignment problem is given by $K^{T}=\left(\alpha_{2}-a_{2}, \alpha_{1}-a_{1}\right) T^{-1}$, where $T=C W, W=\left(\begin{array}{cc}a_{1} & 1 \\ 1 & 0\end{array}\right)$, where $a_{i}(i=1,2)$ are the coefficients of the characteristic polynomial of the matrix $A$. 


$$
\operatorname{det}(\lambda I-A)=\operatorname{det}\left(\begin{array}{cc}
\lambda+0.010 & 0.011 \\
-1.5 & \lambda
\end{array}\right)=\lambda^{2}+0.010 \lambda+0.017
$$

So, $a_{1}=0.010$ and $a_{2}=0.017$.

$$
\begin{aligned}
T & =C W=\left(\begin{array}{cc}
1.44 & -0.144 \\
0 & 2.16
\end{array}\right)\left(\begin{array}{cc}
0.010 & 1 \\
1 & 0
\end{array}\right)=\left(\begin{array}{cc}
-0.13 & 1.44 \\
2.16 & 0
\end{array}\right), \\
T^{-1} & =\left(\begin{array}{cc}
0 & 0.4630 \\
0.6944 & 0.0418
\end{array}\right) .
\end{aligned}
$$

The characteristic roots of $A$ which can also be obtained at the fixed point $\left(x_{*}, y_{*}\right)=(0.46,0.11)$ are $\lambda_{s}=-0.005-$ $0.13 i, \lambda_{u}=-0.005+0.13 i . \alpha_{1}, \alpha_{2}$ are the coefficients of the characteristic polynomial of the matrix $A-B K^{T}$. The assumed characteristic roots $\mu_{1}, \mu_{2}$ are called the adjustment values, that is,

$$
\operatorname{det}\left(s I-\left(A-B K^{T}\right)\right)==s^{2}-\left(\mu_{1}+\mu_{2}\right) s+\mu_{1} \mu_{2} .
$$

The relationships between the roots and the coefficients are obtained as

$$
\begin{aligned}
& \alpha_{1}=-\left(\mu_{1}+\mu_{2}\right), \\
& \alpha_{2}=\mu_{1} \mu_{2} .
\end{aligned}
$$

When $\mu_{1}=1, \alpha_{1}=-1-\alpha_{2}$; when $\mu_{1}=-1, \alpha_{1}=1+\alpha_{2}$; and when $\mu_{1} \mu_{2}=1, \alpha_{2}=1$ according to the range of the values $\alpha_{1}$ and $\alpha_{2}$ determined by equation (22), as in the shaded area in Figure 4.

From Ogata [16] and equation (9), the matrix $K^{T}$ is not unique. As long as the matrix $K^{T}$ is obtained with the values $\alpha_{1}$ and $\alpha_{2}$ in the triangle region MNQ as Figure 4 , it can make the matrix $A-B K^{T}$ be asymptotically stable, that is, the modulus of its eigenvalues is less than 1 , so we can take $\mu_{1}=0, \mu_{2}=\left|\lambda_{s}\right|$. As $\left(\alpha_{1}, \alpha_{2}\right)=\left(-\left|\lambda_{s}\right|, 0\right)$, we can obtain

$$
K^{T}=\left(0-a_{2},-\left|\lambda_{s}\right|-a_{1}\right) T^{-1}=(-0.017,-0.12) .
$$

When $K^{T}$ is found, $\left|K^{T}\left(Z_{i}-Z_{*}(\bar{a})\right)\right|<\delta$ is obtained by $|a-\bar{a}|<\delta$ and equation (12). There is a region whose width is $2 \delta /\left|K^{T}\right|$, and when $Z_{i}$ is in this region, the parameter is controlled; otherwise, the parameter is not controlled. The control rate is given by the following formula:

$$
a-\bar{a}=-K^{T}\left(Z_{i}-Z_{*}(\bar{a})\right) \times u\left(\delta-\left|K^{T}\left(Z_{i}-Z_{*}(\bar{a})\right)\right|\right),
$$

where $u$ is a step function:

$$
u(\alpha)= \begin{cases}0, & \alpha<0 \\ 1, & \alpha>0\end{cases}
$$

As shown in Figure 5, we get the different values in the triangle region of Figure 4 . When we choose $\alpha_{1}=1.75$ and $\alpha_{2}=0.74$, the chaotic motion can be controlled on the period- 1 orbit at $n=5760$ (as shown in Figures 5(a) and 5(b)). When we choose $\alpha_{1}=-0.5$ and $\alpha_{2}=0.6$, the chaotic motion can be controlled

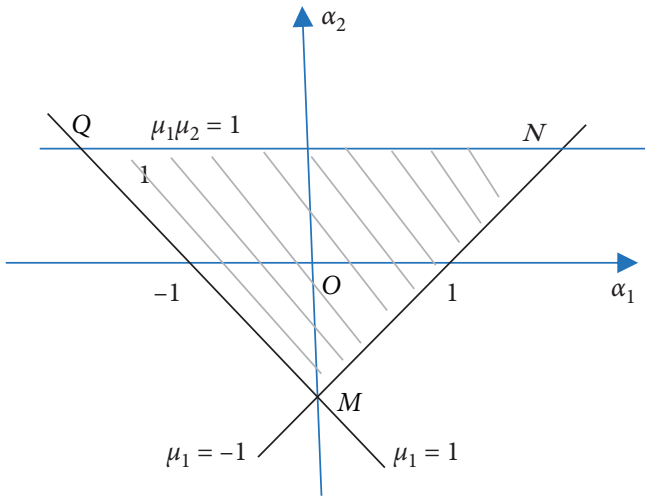

Figure 4: Choice of regulator pole area (triangle region $M N Q$ ).

on the period-1 orbit at $n=3300$ (as shown in Figures 5(c) and 5(d)). When the values of $\alpha_{1}$ and $\alpha_{2}$ are different from the previous values, the number of equation (8) iterations is different to control chaos.

4.2. Control of Period-2 of Hassell Mapping. By iterating equation (8), we can obtain

$$
H\left(H_{a, b}(x, y)\right):\left\{\begin{array}{l}
F(x, y, a) \\
G(x, y, a)
\end{array}\right.
$$

where

$$
\begin{aligned}
F(x, y, a)= & \left(a^{2}(x+\gamma y)(1+x+y)^{-\beta}+\gamma a y\right) \\
& \cdot\left(1+(a x+\gamma a y)(1+x+y)^{-\beta}+y\right)^{-\beta}, \\
G(x, y, a)= & b^{2} x .
\end{aligned}
$$

When $\bar{a}=31$, the points $\left(x_{1}, y_{1}\right)$ and $\left(x_{2}, y_{2}\right)$ of period two are $(0.15,0.26)$ and $(0.34,0.056)$, which are obtained as follows: $F(x, y, a)=x, G(x, y, a)=y$.

According to

$$
\begin{aligned}
& A_{1}=\left(\begin{array}{ll}
\frac{\partial F(x, y, a)}{\partial x} & \frac{\partial F(x, y, a)}{\partial y} \\
\frac{\partial G(x, y, a)}{\partial x} & \frac{\partial G(x, y, a)}{\partial y}
\end{array}\right)_{\left(x_{1}, y_{1}\right)}, \\
& A_{2}=\left(\begin{array}{ll}
\frac{\partial F(x, y, a)}{\partial x} & \frac{\partial F(x, y, a)}{\partial y} \\
\frac{\partial G(x, y, a)}{\partial x} & \frac{\partial G(x, y, a)}{\partial y}
\end{array}\right)_{\left(x_{2}, y_{2}\right)},
\end{aligned}
$$$$
B_{1}=\left(\begin{array}{l}
\frac{\partial F(x, y, a)}{\partial a} \\
\frac{\partial G(x, y, a)}{\partial a}
\end{array}\right)_{\left(x_{1}, y_{1}\right)},
$$

$$
B_{2}=\left(\begin{array}{l}
\frac{\partial F(x, y, a)}{\partial a} \\
\frac{\partial G(x, y, a)}{\partial a}
\end{array}\right)_{\left(x_{2}, y_{2}\right)},
$$




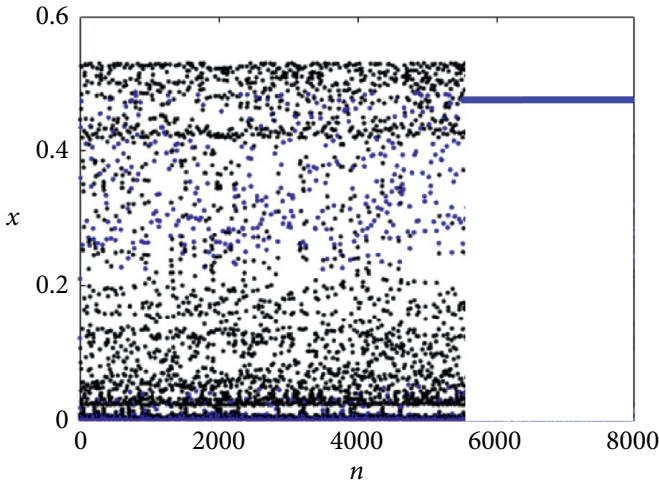

(a)

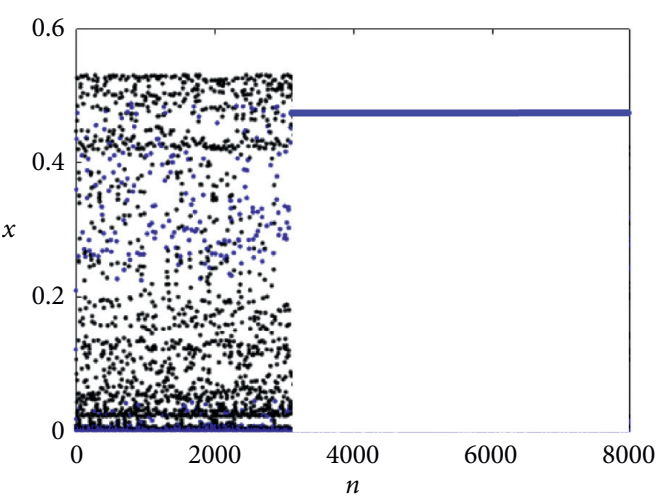

(c)

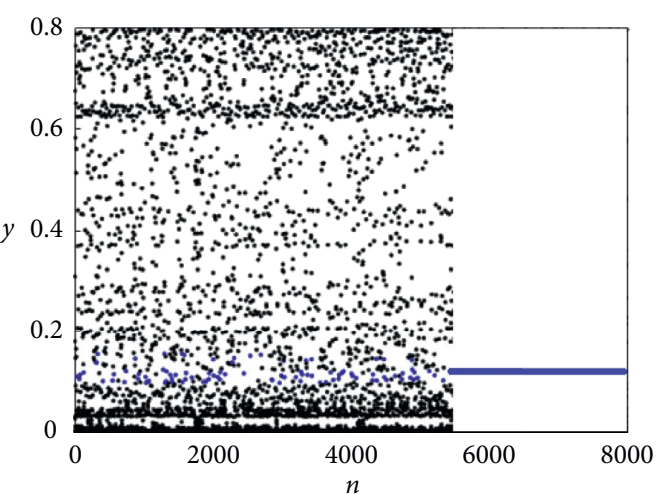

(b)

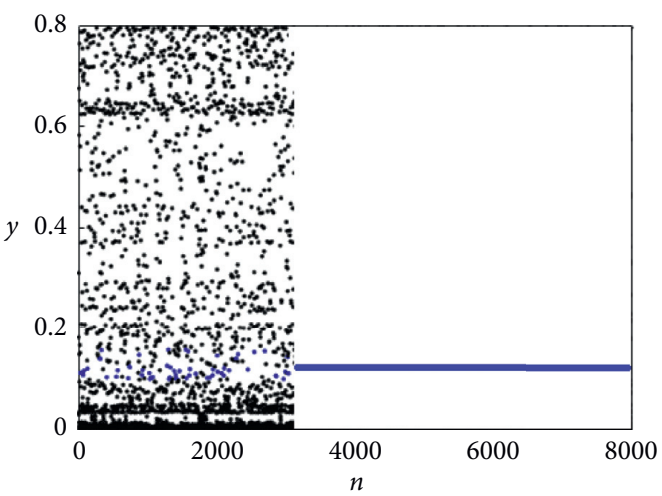

(d)

Figure 5: Control of period-1 of Hassell mapping.

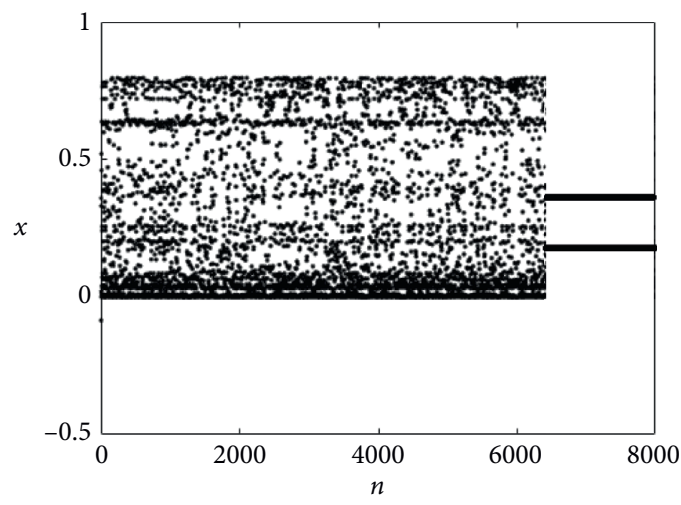

(a)

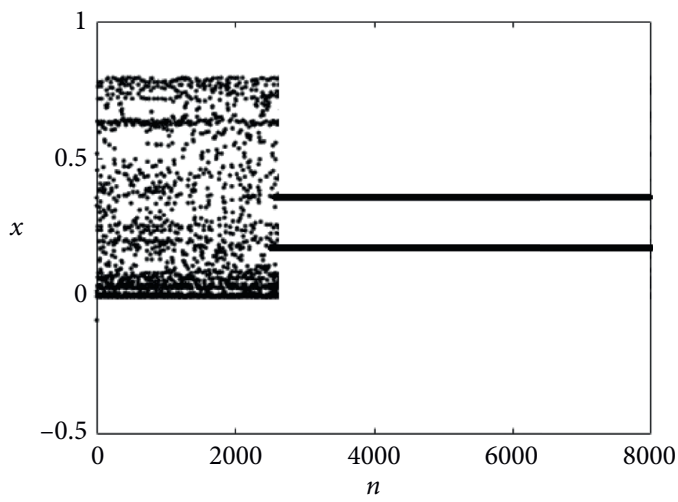

(c)

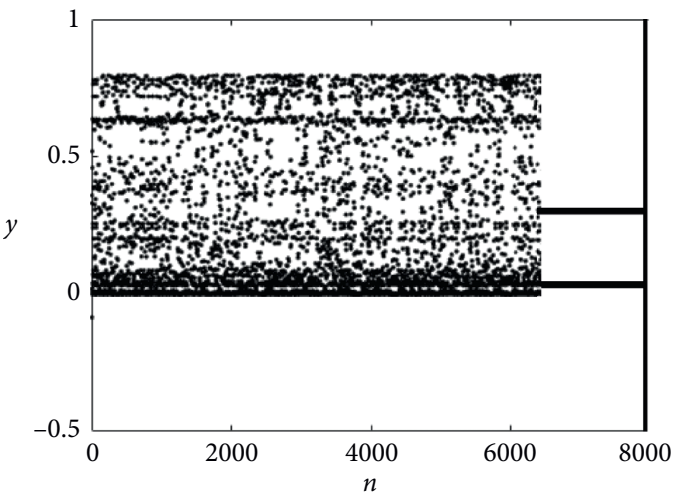

(b)

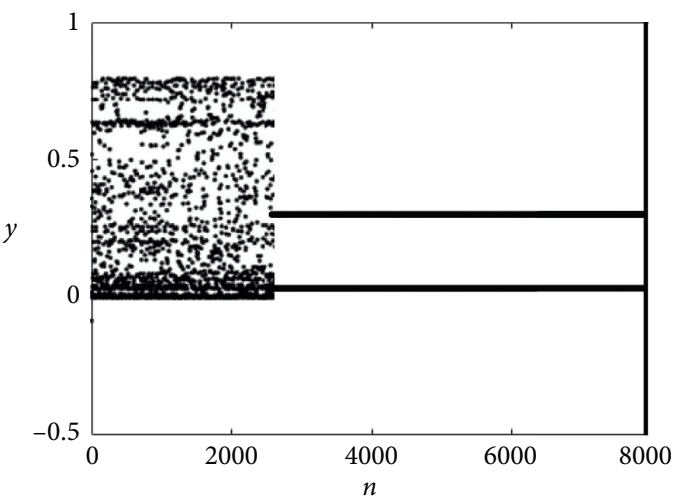

(d)

Figure 6: Control of period-2 of Hassell mapping. 
where

$$
\begin{aligned}
\frac{\partial F(x, y, a)}{\partial x}= & \left(1+(a x+\gamma a y)(1+x+y)^{-\beta}+y\right)^{-\beta} \\
& \cdot\left[a^{2}(1+x+\gamma y)+\left(a^{2}(x+\gamma y)(1+x+y)^{-\beta}+\gamma a y\right)\right. \\
& \cdot(a+a x+\gamma a y)](1+x+y)^{-\beta},
\end{aligned}
$$$$
\begin{aligned}
\frac{\partial F(x, y, a)}{\partial y}= & \left(1+(a x+\gamma a y)(1+x+y)^{-\beta}+y\right)^{-\beta} \\
& \cdot\left[a^{2}(1+x+y)^{-\beta}(1+x+\gamma y)+\gamma a\right. \\
& \left(a^{2}(x+\gamma y)(1+x+y)^{-\beta}+\gamma a y\right) \\
& \left.\left((1+x+y)^{-\beta}(a+a x+\gamma a y)+1\right)\right],
\end{aligned}
$$$$
\frac{\partial G(x, y, a)}{\partial x}=b^{2}
$$$$
\frac{\partial G(x, y, a)}{\partial y}=0
$$$$
\frac{\partial F(x, y, a)}{\partial a}
$$$$
=\left(1+(a x+\gamma a y)(1+x+y)^{-\beta}+y\right)^{-\beta}
$$$$
\cdot\left[2 a(x+\gamma y)(1+x+y)^{-\beta}+\gamma y\right.
$$$$
\frac{\partial G(x, y, a)}{\partial a}=0
$$

Bring $\left(x_{1}, y_{1}\right)=(0.15,0.26)$ and $\left(x_{2}, y_{2}\right)=(0.34,0.056)$ into equation (29)-(33):

$$
\begin{aligned}
& A_{1}=\left(\begin{array}{cc}
-1.54 & -0.98 \\
0 & -3.23
\end{array}\right) 5, \\
& A_{2}=\left(\begin{array}{cc}
0.08 & 0.28 \\
0 & -3.28
\end{array}\right), \\
& B_{1}=\left(\begin{array}{c}
0 \\
0.34
\end{array}\right), \\
& B_{2}=\left(\begin{array}{c}
0 \\
0.44
\end{array}\right) .
\end{aligned}
$$

The controllable matrices are obtained as follows:

$$
\begin{aligned}
& C_{1}=\left(\begin{array}{ll}
B_{1} & A_{1} B_{1}
\end{array}\right)=\left(\begin{array}{cc}
0 & -0.33 \\
0.34 & -1.10
\end{array}\right), \\
& C_{2}=\left(\begin{array}{ll}
B_{2} & A_{2} B_{2}
\end{array}\right)=\left(\begin{array}{cc}
0 & 0.12 \\
0.44 & -1.44
\end{array}\right),
\end{aligned}
$$

and $a_{i}(i=1,2)$ are the coefficients of the characteristic polynomial of the matrix $A_{1}, a_{1}=4.77, a_{2}=4.97$. At the same time, the characteristic roots are $\lambda_{1 s}=-0.54, \lambda_{1 u}=-3.23$.

The solution of the pole assignment problem is given by

$$
K_{1}^{T}=\left(\alpha_{2}-a_{2}, \alpha_{1}-a_{1}\right) T_{1}^{-1},
$$

where $\alpha_{1}$ and $\alpha_{2}$ are the coefficients of the characteristic polynomial of the matrix $A_{1}-B_{1} K_{1}^{T}$. According to the discussion of the values $\alpha_{1}, \alpha_{2}$, we take $\left(\alpha_{1}, \alpha_{2}\right)=\left(-\lambda_{1 s}, 0\right)$, where $T_{1}=C_{1} W_{1}$, so

$$
K_{1}^{T}=\left(0-a_{2},-\lambda_{1 s}-a_{1}\right) T_{1}^{-1}=(-4.97,-4.23),
$$

$a_{i}(i=3,4)$ are the coefficients of the characteristic polynomial of the matrix $A_{2}-B_{2} K_{2}^{T}, a_{3}=3.2, a_{4}=-0.26$, and the characteristic roots are $\lambda_{2 s}=0.08, \lambda_{2 u}=-3.28$. We take $\left(\alpha_{3}, \alpha_{4}\right)=\left(-\lambda_{2 s}, 0\right)$, and we obtain $K_{2}^{T}=(-0.46,4.39)$. The control rate can be given by the following equation:

$$
\begin{gathered}
a-\bar{a}=-K_{i}^{T}\left(Z_{n}-Z(i)(\bar{a})\right) \times u\left(\delta-\left|K_{i}^{T}\left(Z_{n}-Z(i)(\bar{a})\right)\right|\right), \\
i=1,2 .
\end{gathered}
$$

As shown in Figure 6, we get the different values in the triangle region MNQ of Figure 4 . When we choose $\alpha_{1}=$ $-0.37, \alpha_{2}=0.3, \alpha_{3}=-0.25$, and $\alpha_{4}=-0.54$, the chaotic motion can be controlled on the period-1 orbit at $n=6554$ (as shown in Figures 6(a) and 6(b)). When we choose $\alpha_{1}=$ $0.73, \alpha_{4}=0.24, \alpha_{3}=0.34$, and $\alpha_{4}=0.44$, the chaotic motion can be controlled on the period-1 orbit at $n=2700$ (as shown in Figures 6(c) and 6(d)).

\section{Conclusion}

The dynamic complexities of the Hassell recruitment population model have been analyzed. These results demonstrate that the reproductive rate and the resulting population growth can also act as a strong destabilizing factor, leading to rich dynamic behaviors. By improving the method of the OGY, the chaotic control of the Hassell mapping is controlled, the perturbation quantity of the control parameter is selected by the pole configuration method in the linear control theory, and unstable period-1 and unstable period- 2 are controlled to be stable period orbits. At the same time, the adjustment values to be selected are different, and the number of the mapping iterations is different to control chaos. By numerical simulation, the effectiveness of the method is demonstrated. This research can provide theoretical basis and help for the research in biology or ecology. According to the research results, the birth rate $a$, survival rate $b$, or decay coefficient $\beta$ can be properly adjusted to achieve an ecological balance.

\section{Data Availability}

No data were used to support this study.

\section{Conflicts of Interest}

The author declares that there are no conflicts of interest. 


\section{Acknowledgments}

This research was supported by the High Level Scientific Research Project Cultivation Fund of Shandong Women's University; and the National Natural Science Foundation of China (no. 11471259), and the author acknowledges his student Song Xinghao for helping him to do some numerical simulation and manuscript modification.

\section{References}

[1] H. Caswell, Matrix Population Models, Sinauer, Sunderland, MA, USA, 2nd edition, 2001.

[2] I. Ugarcovici and H. Weiss, "Chaotic dynamics of a nonlinear density dependent population model," Nonlinearity, vol. 17, no. 5, pp. 1689-1711, 2004.

[3] M. P. Hassell, "Density-dependence in single-species populations," The Journal of Animal Ecology, vol. 44, no. 1, pp. 283-295, 1975.

[4] A. Wikan and E. Mjølhus, "Over compensatory recruitment and generation delay in discrete age-structured population models," Journal of Mathematical Biology, vol. 35, pp. 195239, 1996.

[5] E. Ott, C. Grebogi, and J. A. Yorke, "Controlling chaos," Physical Review Letters, vol. 64, no. 11, pp. 1196-1199, 1990.

[6] K. Pyragas, "Continuous control of chaos by self-controlling feedback," Physics Letters A, vol. 170, no. 6, pp. 421-428, 1992.

[7] T. Shinbrot, C. Grebogi, J. A. Yorke, and E. Ott, "Using small perturbations to control chaos," Nature, vol. 363, no. 6428, pp. 411-417, 1993.

[8] K. Pyragas, "Predictable chaos in slightly perturbed unpredictable chaotic systems," Physics Letters A, vol. 181, no. 3, pp. 203-210, 1993.

[9] L. Kocarev and U. Parlitz, "General approach for chaotic synchronization with applications to communication," Physical Review Letters, vol. 74, no. 25, pp. 5028-5031, 1995.

[10] F. J. Romeiras, C. Grebogi, E. Ott, and W. P. Dayawansa, "Controlling chaotic dynamical systems," Physica D: Nonlinear Phenomena, vol. 58, pp. 165-192, 1992.

[11] F. Guo, J. Xie, and Le Yuan, "Chaos control of lauwerier mapping," Journal of Southwest Jiaotong University, vol. 49, no. 3, pp. 525-529, 2014.

[12] F. Guo, J. Xie, and Y. Yue, "Chaos control of Lozi mapping," Applied Mechanics and Materials, vol. 509, pp. 23-235, $2014 \mathrm{~b}$.

[13] F. Guo, J. Pan, Y. Mu, and Z. Ji, "The dynamic property and chaos control for a two-degree-of-freedom vibro-impact system," UPB Scientific Bulletin, Series D, vol. 80, no. 1, pp. 1454-2358, 2018.

[14] R. Beaverton and S. Holt, On the Dynamics of Exploited Fish Populations, Vol. 19, Springer, Berlin, Germany, 1957.

[15] M. Hénon, "A two-dimensional mapping with a strange attractor," Communications in Mathematical Physics, vol. 50, no. 1, pp. 69-77, 1976.

[16] K. Ogata, Controlling Engineering, Prentice-Hall, Englewood Cliffs, NJ, USA, 2nd edition, 1990. 